gehen, ich hasse Vorurteile und Stereotype und Schubladen. Und mein Leben ist kein politisches Programm.

Und dann aber auch: Ja, ich habe eine Agenda. Meine Agenda ist das Grundgesetz, im Verbund mit den europäischen und internationalen Grund- und Menschenrechten. Über die lässt sich auch als Richterin eingedenk der richterlichen Zurückhaltung gar nicht genug sprechen.

Heute hielt ich das auch für angemessen. Denn das Verfassungsgericht hat die institutionelle Aufgabe, die sich der djb als Nichtregierungsorganisation, als NGO auf die Fahnen geschrieben hat: Den Grundrechten ernsthaft zur Durchsetzung zu verhelfen. Der djb, so heißt es offiziell, zielt auf die „die Fortentwicklung des Rechts auf allen Gebieten“ zur „Ver- wirklichung der Gleichberechtigung und Gleichstellung der Frau in allen gesellschaftlichen Bereichen “. Er fokussiert also. Der djb ist damit Teil einer offenen Gesellschaft der Verfassungsinterpretation, wie es der Rechtswissenschaftler Peter Häberle einmal wenn auch idealistisch formulierte. Heute ist das eine transnationale Zivilgesellschaft, die sich an Grundund Menschenrechten orientiert, den flachen Versionen entgegentritt, sich nicht einlullen lässt, Substanz einfordert. Das Verfassungsgericht ist mit der Aufgabe des Grundrechtsschutzes folglich nicht allein. Um die Grundrechte müssen sich Viele dauernd kümmern.

Dem Deutschen Juristinnenbund und Ihnen allen wünsche ich dabei viel Erfolg.

\title{
Gleichberechtigung im Bereich der öffentlichen Hand: Anspruch und Wirklichkeit
}

\section{Renate Augstein \\ Ministerialdirigentin, Stellvertretende Leiterin der Abteilung Gleichstellung, Chancengleichheit im Bundesministerium für Fa- milie, Senioren, Frauen und Jugend, Berlin}

Ich freue mich, heute mit Ihnen die Gelegenheit zu haben, die gesetzlichen Instrumente zur Gleichberechtigung im öffentlichen Dienst näher zu betrachten und gemeinsam zu überlegen, in welchen Bereichen sich diese bewährt haben und an welchen Stellen sie weiter geschärft werden müssen. Die öffentliche Hand ist nicht nur durch Artikel 3 Grundgesetz verfassungsrechtlich auf die Gleichberechtigung verpflichtet, sondern ihr kommt auch politisch eine Vorbildfunktion für die gesamte Gesellschaft zu, wenn es um die Herstellung von Gleichberechtigung geht.

Bereits seit 1949 ist in unserem Grundgesetz verankert, dass Männer und Frauen gleichberechtigt sind, und dass niemand wegen seines Geschlechtes benachteiligt oder bevorzugt werden darf. Artikel 3 Grundgesetz wurde später ergänzt um den Staatsauftrag: „Der Staat fördert die tatsächliche Durchsetzung der Gleichberechtigung von Frauen und Männern und wirkt auf die Beseitigung bestehender Nachteile hin". Zur Erfüllung dieses Auftrags wurden verschiedene Instrumente eingesetzt - zum Beispiel das Zweite Gleichberechtigungsgesetz mit dem Frauenförder- und Bundesgremienbesetzungsgesetz 1994, das Bundesgleichstellungsgesetz 2001 und das Allgemeine Gleichbehandlungsgesetz mit der Einrichtung der Antidiskriminierungsstelle des Bundes 2006. Trotzdem haben sich Anspruch und Wirklichkeit in unserem Land nur sehr zögerlich angenähert. Dem von der Bundesregierung 2010 vorgelegten Erfahrungsbericht zum Bundesgleichstellungsgesetz und zum Bundesgremienbesetzungsgesetz lassen sich im Bereich des Anteils von Frauen an den Beschäftigten im öffentlichen Dienst des Bundes und bei der Vereinbarkeit von Familie und Beruf durchaus positive Entwicklungen entnehmen. Der Anteil von Frauen an der Gesamtbeschäftigung in den obersten Bundesbehörden lag 2009 immerhin bei 49 Prozent im Vergleich zu 32 Prozent im Jahr 1986. Familienfreundliche Arbeitsformen wurden in der Bundesverwaltung ausgebaut und werden häufig, inzwischen vermehrt auch von Männern, angenommen.

Gleichzeitig klaffen Anspruch und Wirklichkeit, insbesondere was den Frauenanteil an leitenden Funktionen betrifft, weiterhin auseinander. Frauen sind auf den ersten Führungsebenen in der Privatwirtschaft wie auch im Bereich der öffentlichen Hand nach wie vor selten: Im Jahre 2010 betrug der Anteil von Frauen in den 200 größten deutschen Unternehmen nur 3,2 Prozent bei den Vorständen und 10,6 Prozent bei den Aufsichtsräten. Damit liegt Deutschland im internationalen Vergleich weit zurück. Selbst Schwellenländer wie Brasilien $(6 \%)$ und China $(8 \%)$ weisen einen höheren Frauenanteil in Spitzenfunktionen auf. Bei den Gremien im Einflussbereich des Bundes sieht der Frauenanteil mit knapp 25 Prozent (im Vergleich zu 7\% im Jahr 1991) schon etwas besser aus. Leider gibt es in über zehn Prozent der Gremien immer noch keine einzige Frau. In der Bundesverwaltung liegt der durchschnittliche Anteil von Frauen an Führungspositionen inzwischen bei immerhin 30 Prozent.

Diese Situation ist vor dem Hintergrund der Zukunftsfähigkeit unseres Landes und im Vergleich zu den europäischen Nachbarstaaten weit davon entfernt, zufriedenzustellen. Unter den Vorzeichen des drohenden Führungs- und Fachkräftemangels ist es entscheidend, die nötigen Veränderungsprozesse für eine gleichberechtigte Teilhabe von Frauen in Spitzenfunktionen jetzt anzustoßen. In vielen europäischen Nachbarländern wurde damit längst begonnen und auf EU-Ebene sind bereits unionsweite Maßnahmen geplant. Die Bundesregierung hat sich deshalb die Gleichstellung von Frauen und Männern auf die Fahnen geschrieben. Der Koalitionsvertrag sieht hierzu vor: 


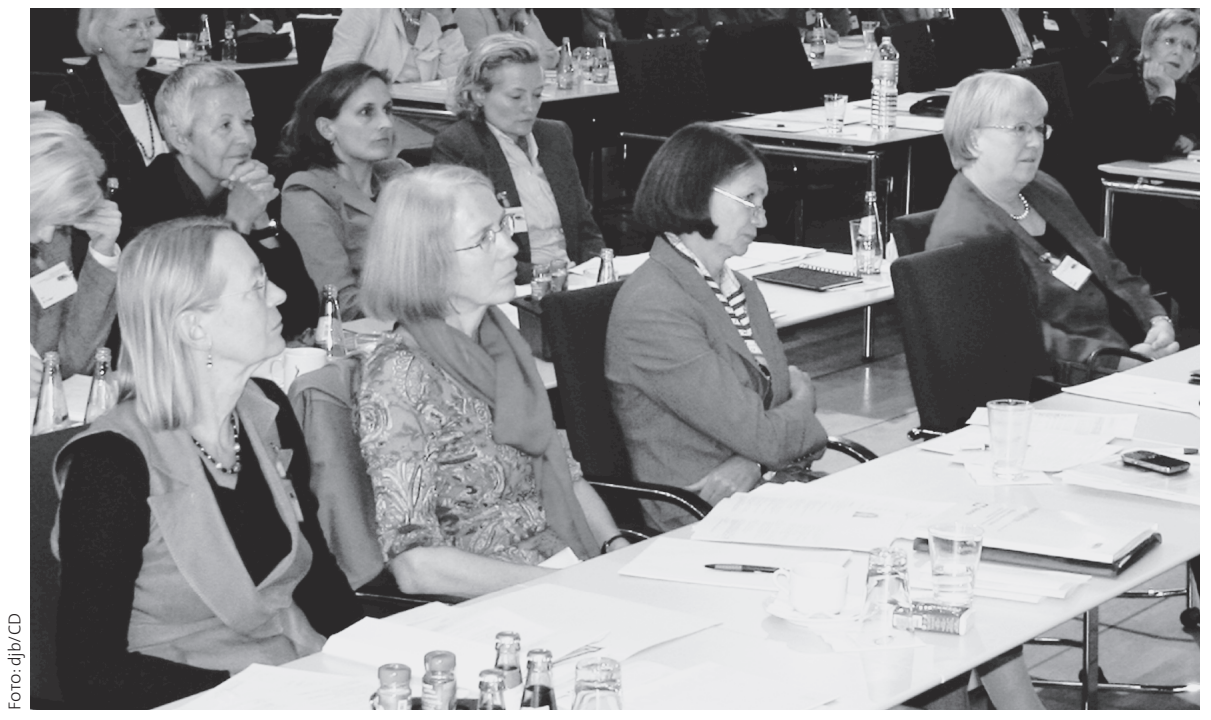

4 Die Tagung am 23. September 2011 im Kongresshotel Potsdam: vorne im Bild Prof. Dr. Sibylle Raasch (Universität Hamburg), Dr. Regine Winter (Richterin am Bundesarbeitsgericht), Jutta Wagner, Renate Augstein (Referatsleiterin im BMFSFJ) (v.I.n.r.).

politisch verfehlt sind. Unternehmen und Verwaltungen sollen sich in ihren unterschiedlichen Ausgangsbedingungen ernst genommen und ihre Vielfalt Berücksichtigung finden. Die selbst gesetzten Ziele müssen veröffentlicht und in Berichten evaluiert werden. Diese Veröffentlichungspflicht sichert die notwendige Transparenz des Verfahrens und schafft Wettbewerb bei den Akteurinnen und „Die Ziele des Bundesgleichstellungsgesetzes und des Bundesgremienbesetzungsgesetzes werden mit Nachdruck verfolgt. Wir werden prüfen, ob und inwieweit die Gesetze geändert und effektiver gestaltet werden müssen. Der Anteil von Frauen in Führungspositionen in der Wirtschaft und im öffentlichen Dienst soll maßgeblich erhöht werden. Dazu wird ein Stufenplan, insbesondere zur Erhöhung des Anteils von Frauen in Vorständen und Aufsichtsräten vorgelegt.“

Im Rahmen dieses Stufenplans beabsichtigt das Bundesfrauenministerium, noch im Jahr 2011 eine gesetzliche Regelung, synchron für Privatwirtschaft und öffentliche Hand, zur Erhöhung des Frauenanteils in Führungspositionen vorzulegen. Der Gesetzentwurf wird Verpflichtungen für Unternehmen und Bundesverwaltung vorschreiben, sich selbst konkrete Ziele zur Erhöhung des Frauenanteils in Führungspositionen zu setzen. Dem liegt der Ansatz zugrunde, dass starre Einheitsquoten für die Gesamtheit der Unternehmen und Verwaltungen ordnungs-

Akteuren. Angesichts des bevorstehenden Fachkräftemangels werden es sich weder private noch öffentliche Arbeitgeber in Zukunft leisten können, gut qualifizierte Frauen durch „gläserne Decken“ zu verlieren. Bei einer Nichteinhaltung der selbst gesetzten Ziele werden Sanktionen greifen, denn wir wissen aus verschiedenen Studien und Erfahrungen anderer Länder, dass reine Quotenregelungen ohne Sanktionen kaum Wirkung entfalten.

Verehrte Mitglieder des djb, wir wollen - auch mit Ihrer Fachkompetenz und Ihrem Engagement - die Chance jetzt nutzen und ein Gesetz zur Erhöhung des Frauenanteils in Führungspositionen auf den Weg bringen, damit Gleichstellung in den Entscheidungszentren unserer Gesellschaft schneller und nachhaltig erreicht wird.

In diesem Sinne wünsche ich uns allen guten Mut, viel Kreativität und Durchsetzungskraft bei der Verfolgung unserer gemeinsamen Ziele - und natürlich eine spannende Fachtagung!

\section{Foren Zugang und Aufstieg im öffentlichen Dienst und in anderen Bereichen}

\section{Dr. Katja Rodi}

Vorsitzende der Kommission Öffentliches Recht, Europa- und Völkerrecht; Wissenschaftliche Mitarbeiterin, Universität Greifswald

\section{Maren Thomsen}

Mitglied der Kommission Öffentliches Recht, Europa- und Völkerrecht; Richterin am Bundesverwaltungsgericht, Richterin am Landesverfassungsgericht Schleswig-Holstein, Leipzig/Kiel

\section{Die Zahlen - keine gleichen Karrierechancen für Frauen im öffentlichen Dienst}

Ziel der Fachtagung war es unter anderem, die Mechanismen und Ergebnisse zu beleuchten, denen sich Frauen beim Zugang und beim Aufstieg im öffentlichen Dienst zu stellen haben, kurzum: zu beleuchten, wieso Frauen nicht die gleichen Karrierechancen im öffentlichen Dienst haben wie Männer. Zugleich sollte auf andere Bereiche geblickt werden, um zu schauen, ob sich hier für Juristinnen Alternativen auftun, aber auch, ob es Übertragungsmöglichkeiten aus diesen Bereichen auf den öffentlichen Dienst im allgemeinen oder die einzelne Frau im öffentlichen Dienst und umgekehrt gibt.

Denn die Zahlen im öffentlichen Dienst sind eindeutig und die sich daraus ergebenden Befunde erschreckend. Sie zeigen, dass es Gründe geben muss, die Frauenkarrieren verhindern und gleichzeitig Männerkarrieren massiv erleichtern. Erst wenn diese Gründe bekannt sind, kann über Abhilfemaßnahmen bis hin zu Gesetzesinitiativen nachgedacht werden. Die 